\title{
Quantifying the adhesive strength between the SARS-CoV-2 S-proteins and human receptor and its effect in therapeutics
}

Mauricio Ponga de la Torre ( $\sim$ mponga@mech.ubc.ca )

The University of British Columbia https://orcid.org/0000-0001-5058-1454

Article

Keywords: SARS-CoV-2, COVID-19, coronavirus, binding affinity, adhesive strength, S-proteins, human receptor

Posted Date: October 19th, 2020

DOI: https://doi.org/10.21203/rs.3.rs-57159/v2

License: (c) (1) This work is licensed under a Creative Commons Attribution 4.0 International License.

Read Full License 


\title{
Quantifying the adhesive strength between the SARS-CoV-2 S-proteins and
}

\author{
Mauricio Ponga* \\ Department of Mechanical Engineering, University of British Columbia, \\ 2054 - 6250 Applied Science Lane, Vancouver, BC, Canada, V6T $1 Z 4$ \\ (Dated: September 25, 2020)
}

\begin{abstract}
The binding affinity and adhesive strength between the spike (S) glycoproteins of the severe acute respiratory syndrome coronavirus 2 (SARS-CoV-2), and the human angiotensin-converting enzyme 2 (ACE2) receptor is computed using molecular dynamics (MD) simulations. The calculations indicate that the binding affinity is $e_{R S}=12.6 \pm 1 \mathrm{kCal} \cdot \mathrm{mol}^{-1}$ with a maximum adhesive force of $\sim 102$ pN. Our analysis suggests that only 27 (13 in S-protein, 14 in ACE2) residues are active during the initial fusion process between the S-protein and ACE2 receptor. With these insights, we investigated the effect of possible therapeutics in the size and wrapping time of virus particles by reducing the binding energy. Our analysis indicates that this energy has to be reduced significantly, around $50 \%$ or more, to block SARS-CoV-2 particles with radius in the order of $R \leq 60 \mathrm{~nm}$. Our study provides concise target residues and target binding energy reduction between S-proteins and receptors for the development of new therapeutics treatments for COVID-19 guided by computational design.
\end{abstract}




\section{SIGNIFICANCE}

$8 \quad$ Viral entry to host cells is a process initiated by binding of proteins in the cell's and virus' surface. 9 The interplay between the virus' (spike) proteins and cells' receptors play a critical role in viral infection. 10 SARS-CoV-2 uses a trimeric spike glycoprotein to attach to human ACE2 receptors to later fuse the virus' 11 envelope with the host cell. Residues in the receptor-binding domain play a critical role in generating short12 range interactions to link these two proteins through adhesive forces. Understanding the energetics, forces, 13 and configurations during this process is crucial to developing efficient treatments based on antibodies and 14 other similar therapeutics. However, the bond's strength between S-proteins and receptors in viruses remain 15 mostly unexplored. Here, we show that the binding energy between the spike proteins and the human ACE2 16 receptor is $12.6 \pm 1 \mathrm{kCal} \cdot \mathrm{mol}^{-1}$ and a maximum force of $\sim 102 \mathrm{pN}$. Molecular simulations allowed us to 17 identify key target residues during the fusion process and elucidate the effectiveness of potential treatments 18 against SARS-CoV-2. Our work suggests that treatments might be more effective in smaller virus particles. 19 In particular, we found the binding energy has to be reduced by a factor of two to effectively block particles 20 with sizes in the range of the SARS-CoV-2. Our work provides a computational framework for the molecular 21 design and assessment of therapeutics against COVID19.

\section{INTRODUCTION}

23 In late 2019 a novel severe acute respiratory syndrome coronavirus (named SARS-CoV-2) was infecting ${ }_{24}$ people in China, causing severe pneumonia [1. Within a few months from the first outbreak, the novel 25 coronavirus created a global pandemic that forced the majority of the world's population under lockdown. ${ }_{26}$ The novel SARS-CoV-2 virus keeps infecting and killing a large number of people around the globe. Thus, ${ }_{27}$ it is imperative to understand and develop therapies that can combat COVID19, the illness caused by the 28 SARS-CoV-2 coronavirus.

29 SARS-CoV-2 belongs to the $\beta$-coronavirus genus 2 and they usually enter to host cells by attaching 30 and fusing to the cell membrane [3]. Cell receptors diffuse across the membrane's external surface to reach 31 proximity with the virus' proteins, binding together for their posterior fusion, promoting membrane bending 32 and virus wrapping until final uptake. Coronaviruses affinity with cell receptors occurs via a transmembrane 33 spike (S) glycoprotein forming homotrimers on the virus' capsid [2, 4]. The S-protein is made of two ${ }_{34}$ functional subunits (S1 and S2) responsible for fusion to the viral-receptor adhesion. Due to their critical 35 role in SARS-CoV-2 infections, S-proteins are the common target for developing antibodies and therapeutics 36 for COVID19.

37 Several works have thus far been focused on characterizing the S-protein and its trimeric structure using 38 cryoEM techniques. For instance, Wrapp et al. [5] have provided a cryoEM structure in the prefusion 39 conformation and have found two states, labeled as up and down, whereby the S1 subunit is exposed and 40 retracted, respectively. The exposed region that links to the human receptor is known as the receptor${ }_{41}$ binding domain (RBD). At the same time, Lan et al. 4 have studied the RBD bounded to the ACE2 and 42 provided a detailed description of the S1 subunit that compose the RBD and its link to the ACE2 receptor. ${ }_{43}$ In particular, they found that the S-protein links to the N-terminus helix of the ACE2 protein serving as an 44 anchor point. Moreover, only a reduced number of residues, in total 20, were in close contact with the ACE2 45 terminus helix and even a smaller portion was within $0.4 \mathrm{~nm}$ from it 4 . This observation suggests that 46 the adhesive forces arise through short-range interactions (e.g., Hydrogen bonds and salt bridges) between 47 these two proteins. Shang et al. 6] provided a structural basis for receptor recognition of SARS-CoV-2. ${ }_{48}$ They found that in addition to the residues in the S1 subunit, many glycans generated links between the 49 two proteins. Understanding the link between the S-protein and ACE2, and in particular, the RBD is key 50 to tackling the pandemic caused by SARS-CoV-2.

51 The importance of the S-protein/ACE2 interface has motivated researchers to explore the phenomenon 52 with both experimental and computational methodologies due to a growing interest in repurposing thera53 peutic to treat COVID19. However, testing the efficacy of these drugs is time consuming and expensive, 54 pushing scientists to develop predictive models based on computational tools to reduce development time. ${ }_{55}$ For instance, Smith et al. [7] have scanned thousands of ligands with molecular dynamics simulations of 
${ }_{56}$ the RBD, and have ranked these ligands based on their affinity. Other studies have focused their attention ${ }_{57}$ on quantifying the S-protein/ACE2 receptor's formation energy, using a full trimeric model and/or a single ${ }_{58}$ S-protein/ACE2 receptor [8 11]. While these studies provide useful information on the compound's forma${ }_{59}$ tion energy, they failed in predicting realistic interaction energies that can be indirectly contrasted with ${ }_{60}$ experimental measurements. This shortcoming is because the adhesive interactions between the S-proteins ${ }_{61}$ and receptors are short-range, and it changes as a function of the separation length. Panda et al. 12 pur62 sued a similar approach to benchmark drugs and antibodies for SARS-CoV-2. These studies focused their ${ }_{63}$ attention on the binding affinity of chemical compounds to reduce the formation energy between SARS${ }_{64} \mathrm{CoV}-2$ S-protein/ACE2 receptor. However, a quantitative evaluation of the binding affinity between the ${ }_{65}$ S-proteins and ACE2 receptors, the adhesive strength of this bond and the chemo-mechanical determinants ${ }_{66}$ controlling coronavirus uptake are still missing. This knowledge gap significantly limits the impact of the ${ }_{67}$ aforementioned investigations and underlines the importance of the proposed study.

68 In this work, we investigate the chemo-mechanical interaction between S-protein and ACE2 receptors, 69 and the resulting implications on the mechanisms for virus uptake. We computed the binding affinity and 70 adhesive force between S-protein and ACE2 receptors and analyzed the residues in contact during the bond71 breaking process. Surprisingly, our results indicate that the residues in contact change as the two proteins 72 were pulled apart, elucidating target points to develop new therapeutics. With these findings, we investigated ${ }_{73}$ SARS-CoV-2 uptake to predicted the effects of the binding affinity perturbations on the uptake kinematics.

\section{RESULTS AND DISCUSSIONS}

75 After performing umbrella sampling simulations on the S-proteins/ACE2 receptor configuration, we inves76 tigated the potential of mean force (PMF) evolution as a function of the pulling distance, i.e., the reaction 77 coordinate $(\lambda)$ (see Figure 1(a) for a schematics). Figure 1(b) shows the results for the single and full S78 protein/ACE2 receptor configuration. Focusing our attention on the full trimeric protein, we observed that, 79 initially, the evolution of the PMF shows a metastable and a global minimum between $\lambda=0-0.4 \mathrm{~nm}$. so These configurations were separated by a small barrier of $\sim 1.5 \mathrm{kCal} \cdot \mathrm{mol}^{-1}$. For $0.4 \leq \lambda \leq 1.4 \mathrm{~nm}$, the 81 PMF's evolution shows almost a linear behavior with the reaction coordinate up to approximately $\lambda=1.4$ $82 \mathrm{~nm}$, where the PMF reached $\sim 12.6 \pm 1 \mathrm{kCal} \cdot \mathrm{mol}^{-1}$. Thereafter, the PMF changes slopes and tends to s3 plateau around $\sim 20 \pm 1 \mathrm{kCal} \cdot \mathrm{mol}^{-1}$ at the end of the sampling, when $\lambda \geq 4 \mathrm{~nm}$. The maximum error in 84 the measure of the PMF is approximately $\sim \pm 1 \mathrm{kCal} \cdot \mathrm{mol}^{-1}$.

85 The PMF's change at around $\lambda=\sim 1.5 \mathrm{~nm}$ indicates that at this point, all van der Waals interactions ${ }_{86}$ are off between the two proteins, as shown with the change of slope in the plot. This was also confirmed by ${ }_{87}$ analyzing the residues in contact (below). Remarkably, the position where the change of slope happens is very 88 close to the selected cutoff of the van der Waals interaction set up in our model. The remaining interactions 89 appear due to long-range electrostatic forces that are in the model. We take the curve's inflection point as 90 the value of the adhesive strength that characterizes the link between the S-protein/ACE2 receptor. The ${ }_{91}$ result obtained with a single S-protein/ACE2 receptor leads to approximately the same free energy but a 92 slightly different path. The binding affinity is estimated to be $e_{R S}=12.6 \pm 1 \mathrm{kCal} \cdot \mathrm{mol}^{-1}$ for the full trimeric ${ }_{93}$ model, and $e_{R S}=12.55 \pm 0.7 \mathrm{kCal} \cdot \mathrm{mol}^{-1}$ for the single S-protein/ACE2 receptor indicated with stars in the 94 plot.

${ }_{95}$ The force separation $\mathbf{F}$ between the S-protein and ACE2 receptor is obtained from the relation $\mathbf{F}=$ ${ }_{96} d e_{R S} / d \lambda$, hence the slope of the PMF curve in Figure 1(b). The force evolution is shown in Figure 1)(c) for 97 both models. We observed that the force builds up to a maximum of $\mathbf{F}_{\max } \sim 102 \mathrm{pN}$ denoting the rupture 98 force between the S-protein/ACE2 receptor bond, in the order of magnitude expected for adhesion in cells 99 [13. Thereafter, the force drops significantly due to lack of contact between the residues.

100 The binding energy between the S-proteins/ACE2 receptor can be used to compute the dissociation con101 stant $K_{D}=1.32 \mathrm{nM}$ (see Supplementary information (SI) for calculation of the constant). Recent 102 works have estimated the dissociation constant of the SARS-CoV-2 virus in experimental setups, obtaining 103 values between $1.2 \pm 0.1 \mathrm{nM}$ to $4.674 \mathrm{nM}$ [2, 4]. The almost five-fold discrepancy range among previous 104 measurements underlines the difficulty in obtaining accurate experimental data and, also, remarks the po105 tential impact of our computational method. Considering that our model is limited to only a small portion 
a

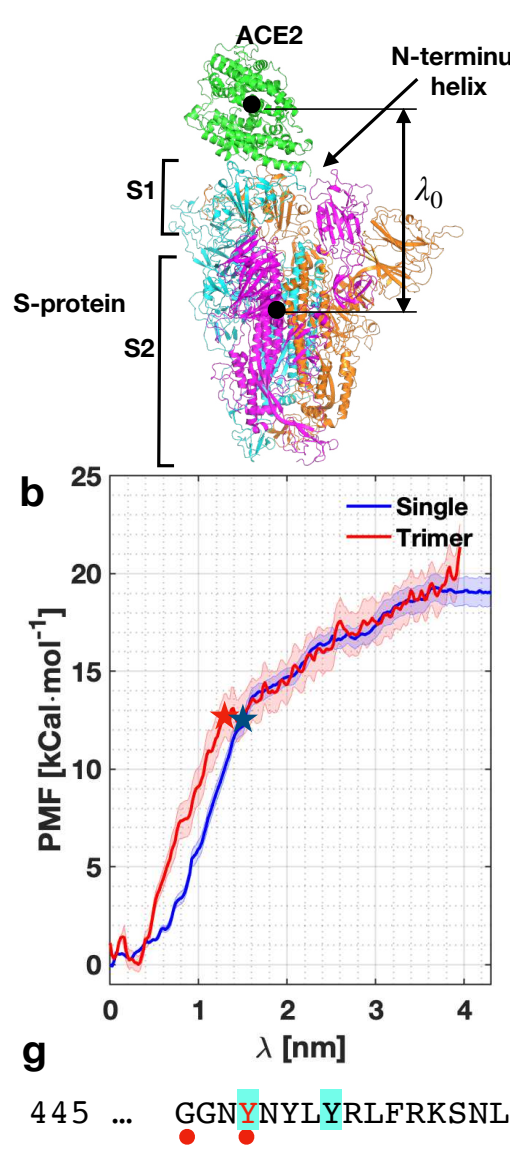

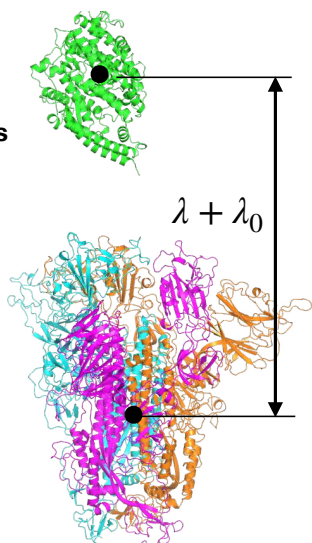

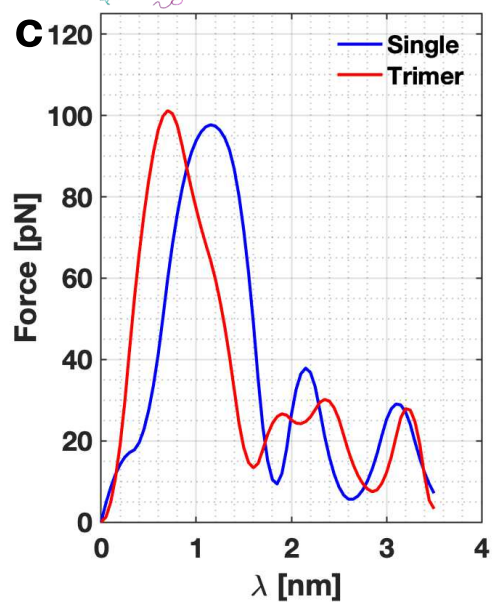

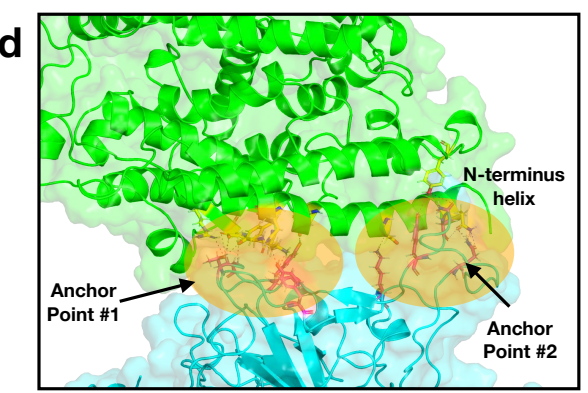

e

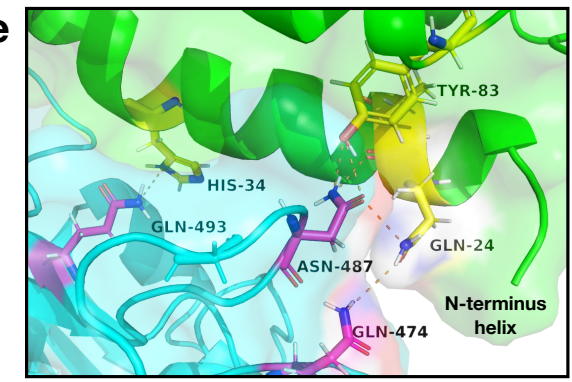

f

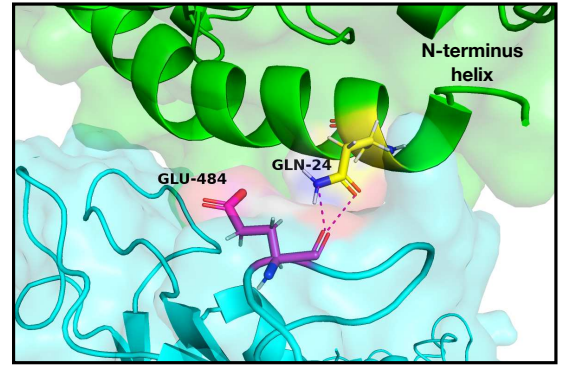

FIG. 1: Evolution of the PMF, force, and residues with $\lambda$. (a) Schematics of the setup. (b) and (c)

Evolution of the PMF and force as a function of $\lambda$ for a single S-protein/ACE2 receptor (blue) and for the trimeric protein (red). Shaded area in (b) represents the error band for both simulations. The stars indicate the moment when detachment has happened. (d) View of the two anchor points between S-protein/ACE2 receptor when $\lambda=0.3 \mathrm{~nm}$. (e) Interacting residues for $\lambda=0.8 \mathrm{~nm}$ and (f) $\lambda=1.4 \mathrm{~nm}$. $(\mathrm{g}$ ) List of residues that are active during the pulling simulation in the RBD. Letters in red, cyan background, bold and underlined correspond to residues in contact at $\lambda=0.3,0.4,0.8,1.4 \mathrm{~nm}$, respectively. Red dots indicate residues identified experimentally by Lan et al. 4. Residue K-417 has been omitted in the sequence for shortness.

106 of the real virus/cell receptor, the agreement between the simulations and experiments is remarkable and 107 gives confidence in our computational approach.

109 We now focus our attention on the molecular interactions between S-protein and ACE2 receptor as a ${ }_{110}$ function of the reaction coordinate $\lambda$. The analysis of the interactions was performed with the full model at ${ }_{111}$ coordinates $\lambda=\sim 0.3,0.4,0.8,1.4,1.6 \mathrm{~nm}$. Using the most typical cluster configurations (see Methods), ${ }_{112}$ we first obtained the interface residues between the two molecules and performed a contact analysis between 
113 that group. We disregarded all residues whose distance was more than $0.4 \mathrm{~nm}$. As expected, the number of 114 interactions decreased when $\lambda$ increased.

115 First, we analyze the configuration with minimum free energy in our simulations, corresponding to $\lambda=0.3$ $116 \mathrm{~nm}$, shown in Figure $1(\mathrm{~d})$. The S-protein anchors from two locations; namely, the ends of the N-terminus 117 helix in the ACE2 receptor and it could reach the helix on top if it, as shown in Figure 1(d). We observed 118 that the interacting residues in the S-protein were located between positions 417-505 of the sequence, namely 119 residues K-417, Y-449, Q-474, N-487, Y-489, G-496, T-500, G-502, Y-505, as shown in Figure 1(g) -using 120 a one-letter sequence- with red letters (see also Figure SI1, Table SI1 and VideoSI1 in the SI ). These ${ }_{121}$ residues linked to residues Q-24, D-30, E-37, Y-41, Q-42, Y-83, K-353, G-354, D-355, R-357 in the ACE2 122 receptor. When $\lambda=0.4 \mathrm{~nm}$, ten residues were active - five were the same- (denoted with a cyan background ${ }_{123}$ in Figure $1(\mathrm{~g})$ ) with a graphical representation in Figure SI1. When $\lambda=0.8$ nm, we observed interactions 124 between the terminus helix and the one on top, as shown in Figure 1(e) involving Q-493, N-487, and Q-474 125 in the S-protein (bold letters in Figure 1(g)), and Q-24, H-34, Y-83 in the ACE2 receptor. These residues ${ }_{126}$ were the most persistent ones, generating stronger links than other residues through the bond-breaking ${ }_{127}$ simulation. Thus, these residues can be targeted in new therapeutics strategies in COVID19. For $\lambda=1.4$ ${ }_{128} \mathrm{~nm}$, we found that only two residues were interacting, namely E-484 and Q-24 in the S-protein and ACE2 129 receptor, respectively (underlined in Figure 1 $(\mathrm{g})$ ). Figure 1(f) shows the links between these residues (see ${ }_{130}$ SI VideoSI2). For $\lambda \geq 1.6 \mathrm{~nm}$, no contacts were found.

${ }_{131}$ From the 27 residues in the RBD, our simulations indicate that 13 were active during the pulling simulation. ${ }_{132}$ In particular, we identified 13 unique residues that were active in the S-protein, and 14 in the ACE2 (see ${ }_{133}$ Table SI1). These residues are the same to the ones identified by Lan et al. [4] in their cryoEM analysis, 134 with the exception of G-446, N-501 and Q-493. However, we did identify G-502 and Q-498, which are very 135 close to the previously mentioned residues. This remarkable agreement gives confidence to our approach and 136 simulations.

\section{B. Uptake modeling and the effect of therapeutics}

${ }_{138}$ We now analyze the effect of the binding affinity in the endocytosis of the virus in cells. We recur to the 139 chemo-mechanical model developed by Gao et al. 14. The model considers the bending energy of the cell 140 membrane, the release of chemical energy during the fusion of S-protein and receptor, the configurational ${ }_{141}$ entropy, and the ratio between receptor and $\mathrm{S}$-protein density $\tilde{\xi}=\xi_{R} / \xi_{S}$ (see Methods). Also, the model ${ }_{142}$ needs specific parameters that are characteristic for each virus. We discuss these parameters - which are 143 summarized in Table I- for SARS-CoV-2 in Methods.

${ }_{144}$ We found that the model predicts a minimum radius of $R_{\min }=27 \mathrm{~nm}$ with an optimum of $R_{o p}=30$ ${ }_{145} \mathrm{~nm}$ at which the uptake time is minimum (around $t_{w}^{\min } \sim 3 \mathrm{~s}$, see Figure SI2) for $\tilde{\xi}=0.1$. Particles ${ }_{146}$ below $R_{\min }$ cannot be wrapped because the uptake is not energetically allowed. For smaller values of $\tilde{\xi}$, we 147 found that the minimum radius increases, in particular, for a $\widetilde{\xi}=0.0001$ the minimum and optimal radius ${ }_{148}$ are $R_{\min }=34 \mathrm{~nm}$, and $R_{o p}=38.5 \mathrm{~nm}$, respectively with a minimum wrapping time $t_{w}^{\text {min }} \sim 15000 \mathrm{~s}$, see ${ }_{149}$ Figure SI2. These trends are in agreement with Gao's findings 14. The predicted minimum radius of $150 \sim 30 \mathrm{~nm}$ is in close agreement with experimental observations of SARS-CoV-2 particle size [1, 15] where 151 the minimum particle radius was $30 \mathrm{~nm}$. This constraint could be implicit in the molecular architecture of 152 the virus. However, our predictions suggest that viral particles that are smaller than $\sim 30 \mathrm{~nm}$ cannot be 153 uptaken, hence preventing their reproduction inside the host cell. Moreover, the predicted optimal radius of ${ }_{154} 30-40 \mathrm{~nm}$ is close to the average particle size measured experimentally of $R_{\text {ave }}=50 \mathrm{~nm}[1,15$.

${ }_{155}$ Current attempts to treat COVID19 aim to repurposing therapeutics drugs and antibodies to bind between ${ }_{156} \mathrm{~S}$-proteins and ACE2 receptors, thereby reducing $e_{R S}$ [7, 12. Next, we provide an estimation of the reduction ${ }_{157}$ of $e_{R S}$ needed to increment $R_{\min }$ above the radius of SARS-CoV-2 particles (i.e., to stop particles from being 158 uptaken). To this end, we modified the S-protein/receptor binding affinity $e_{R S}^{*}=k e_{R S}$, where $k \in[0,1]$ is a 159 reduction factor giving no affinity for $k=0$ and full affinity when $k=1$. Thus, $k$ represents the effectiveness 160 of the treatment in reducing the binding affinity between S-proteins and ACE2 receptors.

${ }_{161}$ Figure 2 shows the relation between $R_{\min }$ and $k$ for various $\tilde{\xi}$. The green region identifies all particles 162 radii for which virus uptake is always permitted independently of any reduction in binding affinity $(k)$. 
TABLE I: Parameters used to model the endocytosis process in an infinite membrane. The reference temperature was taken as $T=310.15 \mathrm{~K} . R$ is the radius of SARS-CoV-2 particles, $\xi_{S}$ is the density of spike proteins, $D$ is the diffusivity of receptors, $B$ is the bending modulus of lipid bilayer, $e_{R S}$ is the binding affinity, and $\tilde{\xi}$ is the ration between receptor and S-protein density.

\begin{tabular}{cccccc}
\hline$R[\mathrm{~nm}]$ & $\xi_{S}\left[\mu \mathrm{m}^{-2}\right]$ & $D\left[\mu \mathrm{m}^{2} \cdot \mathrm{s}^{-1}\right]$ & $B\left[\mathrm{kCal} \cdot \mathrm{mol}^{-1}\right]$ & $e_{R S}\left[\mathrm{kCal} \cdot \mathrm{mol}^{-1}\right]$ & $\tilde{\xi}=\frac{\xi_{R}}{\xi_{S}}$ \\
\hline $30-70$ & 2930 & 0.01 & 12.3 & 12.6 & $10^{-1}-10^{-4}$ \\
\hline
\end{tabular}

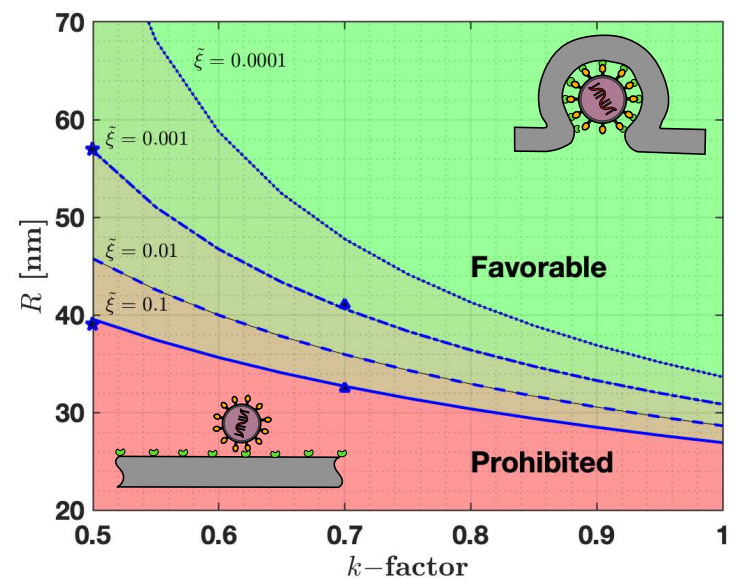

FIG. 2: Effect of reduced binding affinity ( $k$-factor) in the size of particles that can be wrapped to the cell membrane. Four receptor densities were used, namely $\tilde{\xi}=0.1$ (solid line), $\tilde{\xi}=0.01$ (dashed line), $\tilde{\xi}=0.001$ (dashed-dotted line), $\tilde{\xi}=0.0001$ (dotted line). Prohibited particles are in the red zone, while favorable particles are shown with in green. In between these zones, particle will be blocked depending on the relative receptor/S-proteins density $\tilde{\xi}$.

${ }_{163}$ The red region identifies particles whose radii are not permitted to be uptaken due to excessive bending 164 energy. Between these regions, virus uptake is controlled by $\tilde{\xi}$. For instance, if the binding affinity between 165 S-proteins and ACE2 receptors is reduced by $30 \%(\mathrm{k}=0.7)$, the minimum radius of particles that can be ${ }_{166}$ uptaken increases by $21.5 \%$ for $\tilde{\xi}=0.1\left(R_{\min }^{k=0.7}=33 \mathrm{~nm}\right)$, and by $32 \%$ for $\tilde{\xi}=0.001\left(R_{\min }^{k=0.7}=41 \mathrm{~nm}\right.$, 167 blue triangles in Figure 2). Another important aspect is that the wrapping time required for final uptake 168 increases about 50 to $100 \%$ for these cases, respectively (see Figure SI3). This increment in the time needed 169 for final uptake might be critical in some cases since it might give the immune system extra time to combat 170 the infection, thus illustrating the effect of the treatment.

${ }_{171}$ If we reduce the binding affinity by $50 \%(k=0.5)$, the minimum radius increases by $47 \%$ when $\tilde{\xi}=0.1$ ${ }_{172}\left(R_{\min }^{k=0.5}=39 \mathrm{~nm}\right)$, and by $84 \%$ when $\tilde{\xi}=0.001\left(R_{\min }^{k=0.5}=57 \mathrm{~nm}\right.$, blue stars in Figure 2). The wrapping 173 time also increases by $150 \%$ to $425 \%$ with respect to the case when $k=0$ (see Figure SI3) and allowing 174 additional time to stop infection in cells. Finally, our work provides a rough estimation of how much binding 175 affinity has to be reduced to provide effective treatments. Our analysis indicates that a $50 \%$ reduction in ${ }_{176} e_{R S}$ might stop the adhesion of SARS-CoV-2 particles with $R \leq 60 \mathrm{~nm}$. Moreover, we predict that smaller ${ }_{177}$ particles $(R \leq 60 \mathrm{~nm})$ are more suitable to be blocked in comparison with larger ones, based on bending 178 energy analysis. 
180 Our study reports the first attempt (to the author's best knowledge) to evaluate the binding affinity and 181 bond-breaking force between SARS-CoV-2 spike proteins and ACE2 receptors via computational analysis 182 using an all-atom MD model. Our estimation of $e_{R S}=12.6 \pm 1 \mathrm{kCal} \cdot \mathrm{mol}^{-1}$ gives a dissociation constant ${ }_{183}$ of $K_{D}=1.3 \mathrm{nM}$, which is in close agreement with experimental measurements $\left(K_{D}=1.4-44 \mathrm{nM}\right)$. ${ }_{184}$ Furthermore, our analysis shows that only a reduced fraction (about 13) of the residues in the RBD interact 185 during the protein adhesion. These residues play a critical role in the adhesion of the S-protein/ACE2 186 receptors, and can be used as a target for therapeutic strategies to prevent virus uptake in human cells. ${ }_{187}$ Finally, using a mechanistic model to analyze virus uptake, we concluded that S-proteins/ACE2 receptor's 188 binding has to be reduced at least by $50 \%$ to block the uptake of SARS-CoV-2 particles having radius 189 between 30 to $70 \mathrm{~nm}$. However, according to our analysis, the effectiveness of these strategies is strongly 190 dependent on particle size and receptor density. Hence, such treatments might be more effective in blocking 191 only a portion of the SARS-CoV-2 particles leaving others unaffected.

\section{ACKNOWLEDGEMENTS}

193 We thank Dr. Mattia Bacca and Dr. Daniel Aguilar-Hidalgo (University of British Columbia) for useful 194 discussions and comments on the manuscript. We gratefully acknowledge the support from the Natural 195 Sciences and Engineering Research Council of Canada (NSERC) through the Discovery Grant under Award 196 Application Number RGPIN-2016-06114, the New Frontier in Research Fund Exploration program through 197 the award NFRFE-2019-01095 and the support of Compute Canada and the Advanced Research Computing 198 (ARC) at the University of British Columbia.

\section{A. Molecular dynamics simulations, Umbrella sampling and characterization}

201 Molecular dynamics simulations were performed with the GROMACS software [16 18. The molecular 202 geometry was taken from different sources, including the protein data base models 6LZG for a single S${ }_{203}$ protein [19], and the PDB file 6VYB full trimeric model [2]. In addition -since we started our simulations 204 before the PDB models were available - we used model provided by Smith et al. [7] using the sequences 205 available online (NCBI Reference: YP_009724390.1) for the SARS-CoV S-protein's crystal structure and the 206 ACE2 receptor was generated using the PDB 2AJF file. While the actual numbers vary slightly, the trends 207 are the same regardless the geometry. The model was loaded into GROMACS, where it was solvated in 208 water using the TIP3P model to achieve a density of approximately $\rho=1000 \mathrm{Kg} \cdot \mathrm{m}^{-3}$. In order to allow for 209 sufficient space for the pulling simulations, we generated computational cells with more than $1 \mathrm{~nm}$ between 210 the proteins and end of the cells, and sufficient space on top to perform the pulling simulations. The biggest ${ }_{211}$ cell size has dimensions of $\sim 13.84 \times 14.99 \times 21.47(\mathrm{~nm})$. After adding the solvent, the system had a non-zero 212 charge and sodium $\left(\mathrm{Na}^{+}\right)$ions were added as needed to equilibrate in all samples. All interatomic forces ${ }_{213}$ were computed with the CHARMM force-field [20. The biggest cell had 648,265 atoms including proteins 214 and solvent.

215 The solvated system was initially subjected to an energy minimization using a non-linear conjugate gra216 dient. The forces were minimized with a convergence criterion of $1000 \mathrm{~kJ} \cdot \mathrm{mol}^{-1} \cdot \mathrm{nm}^{-1}$. After the system ${ }_{217}$ was relaxed, it was subjected to an NPT ensemble for $1 \mathrm{~ns}$ using an initial temperature of $T=310.15 \mathrm{~K}$ 218 imposed with a Berendsen thermostat [21. Pressure was controlled to 1 bar using a Berendsen barostat. ${ }_{219}$ The timestep was set to $\Delta t=2 \mathrm{fs}$. For all simulations carried in this work, short-range interactions were 220 treated with a smooth force-switch cutoff of $r=1.2 \mathrm{~nm}$, and long-range electrostatics were treated using the ${ }_{221}$ Particle-Mesh-Ewald (PME) formalism, implemented in GROMACS 22. Hydrogen-bonds were restrained 222 with the LINCS algorithm [23]. 
${ }_{223}$ In order to compute the binding affinity between the S-proteins/ACE2 receptor, we used a combination of 224 pulling simulations with umbrella sampling. The initial configuration of the S-proteins/ACE2 was subjected 225 to a pulling simulation to generate the necessary configurations to perform an umbrella sampling. The pulling 226 simulation was performed with an optimized spring constant of $K=1300 \mathrm{~kJ} \cdot \mathrm{mol}^{-1} \cdot \mathrm{nm}^{-2}$. This optimized 227 spring constant was obtained by performing multiple umbrella samplings on the single S-protein-ACE2 228 receptor with spring constants in the range of $K=750-2000 \mathrm{~kJ} \cdot \mathrm{mol}^{-1} \cdot \mathrm{nm}^{-2}$, and optimizing the value 229 using a quadratic fitting. Values obtained for different spring constants are shown in the SI. The pulling 230 rate was set to $v_{z}=5 \mathrm{~nm} \cdot \mathrm{ns}^{-1}$ along the $\mathrm{z}$-direction and sampling simulations were run for a total time of ${ }_{231} t=10 \mathrm{~ns}$. The pulling direction was set such that the S-protein and ACE2 receptor were pulled apart from 232 each other. The configurations generated along the pulling simulations were systematically used to generate 233 trajectories for the umbrella sampling, described below.

${ }_{234}$ Umbrella simulations were performed for configurations separated about $\Delta \lambda=0.1 \mathrm{~nm}$ from the reference ${ }_{235}$ configuration. Each configuration was constrained with a spring constant of $K=1300 \mathrm{~kJ} \cdot \mathrm{mol}^{-1} \cdot \mathrm{nm}^{-2}$ and ${ }_{236}$ run for $t=10 \mathrm{~ns}$. These simulations provided enough sampling to obtain the potential of mean force along the ${ }_{237}$ reaction path to display the evolution of the free energy of the system. The PMF was then estimated using the ${ }_{238}$ Weighted Histogram Analysis Method (WHAM) 24 using a bootstrap analysis to estimate the uncertainty 239 in the PMF. We used 100 different measures, using 200 binning spaces along the reaction coordinate $\lambda$. We 240 performed several simulations with the backbone of the proteins fixed and without any fix conditions. We 241 found that simulations without fixing the backbone produce excessive elasticity in the proteins and lead to 242 higher free energies.

243 Analysis of the atomistic configurations was performed with GROMACS cluster analysis tool. We scanned 244 the configurations with a root mean squared displacement between the range of $0.15-0.25 \mathrm{~nm}$ [25]. The 245 cluster analysis yielded between three to six cluster for the analyzed configurations. In all cases shown, the 246 most populated cluster was used when analyzing the configurations. The configurations were then analyzed 247 with the software Pymol.

\section{B. Mechanistic model of endocytosis}

249 Gao et al. 14 developed a model considering a spherical particle being attached to an infinite membrane. ${ }_{250}$ The fusion of the particle with the membrane is driven due a release of the binding energy -computed 251 above- when the S-protein and receptors are linked. It is assumed that the membrane has an equilibrium 252 concentration of receptors, $\xi_{R}$, and when the particle attaches, this concentration changes with time, e.g., ${ }_{253} \xi(s, t), s$ being the arc-length. In particular, when a particle is attached to the membrane, the density ${ }_{254}$ of receptors, $\xi(s, t)$, matches to the density of spike proteins, $\xi_{S}$, and far away tends to the equilibrium 255 concentration. Considering the bending energy of a lipid bilayer - characterized through its bending modulus ${ }_{256} B$ and curvature $\kappa=\frac{2}{R}-$, and the binding energy between S-proteins and receptors, $\left(e_{R S}\right)$ one can write 257 down the following free-energy for the endocytosis process as

$$
F(t)=k_{B} T\left[\int_{0}^{a(t)}\left(\frac{1}{2} B \kappa^{2}-\xi_{S} e_{R S}+\xi_{S} \ln \left(\frac{\xi_{S}}{\xi_{R}}\right)\right) d s+\int_{a(t)}^{\infty} \xi \ln \frac{\xi}{\xi_{R}}\right] .
$$

${ }_{258} \mathrm{In} \mathrm{Eq}$. 1. $k_{B} T$ is the thermodynamic factor with $k_{B}$ and $T$ denoting the Boltzmann's constant and the 259 absolute temperature, respectively. By requiring that the rate of free energy reduction gained in the wrapping 260 process exactly balance the rate of energy dissipation consuming during the transport, Gao et al. found that 261 there exists an optimal wrapping radius of the particles and a minimum radius below the particle cannot be 262 wrapped. The wrapping time can be found as

$$
t_{w}=\left(\frac{R}{\alpha \sqrt{D}}\right)^{2}
$$

263 where $R$ is the radius of the particle, $\alpha$ is the speed factor $(\alpha>0)$, and $D$ is the diffusivity of the receptors 264 in the membrane. The speed factor is found by solving the rate equation 


$$
e_{R S}-\frac{1}{2} \frac{B \kappa^{2}}{\xi_{S}}-f(\alpha)+\ln f(\alpha)+1=0
$$

with

$$
f(\alpha)=\tilde{\xi}+\frac{\alpha^{2}(1-\tilde{\xi}) E_{1}\left(\alpha^{2}\right)}{\alpha^{2} E_{1}\left(\alpha^{2}\right)-\exp \left(-\alpha^{2}\right)} .
$$

${ }_{266}$ The parameter $\tilde{\xi}=\frac{\xi_{R}}{\xi_{S}}$ defines the ratio between equilibrium receptor density in the membrane and the ${ }_{267} \mathrm{~S}$-porteins in the virus particle. In the above expression, $E_{1}$ is the exponent integral function defined as

$$
E_{1}(x)=\int_{x}^{\infty} \frac{\exp (-u)}{u} d u
$$

The model predicts a minimum radius for spherical particle given by

$$
R_{\text {min }}=\left(\frac{2 B}{\xi_{S}\left[e_{R S}-\tilde{\xi}+\log \tilde{\xi}+1\right]}\right)^{1 / 2},
$$

269 and an optimal radius that is determined numerically. Gao et al. determined the wrapping time as a function 270 of the particle radius as well as the optimal particle radius numerically.

\section{Parameters determination}

272 SARS-CoV-2 virus particles of sizes between $R=30-70 \mathrm{~nm}$ have been reported [1, 15. This range 273 indicates a wide range of particles, with an average size of $R_{\text {ave }}=50 \mathrm{~nm}$. Microscopic images indicate 274 that around $17 \pm 2$ spike proteins in the circumference of the virus. An elementary analysis indicates that 275 the density of spike proteins in the novel SARS-CoV-2 virus must vary between $\xi_{S}=2280-3660 \mu \mathrm{m}^{-2}$ 276 (about $\sim 90$ spike proteins in the surface) when the average radius is taken. The computed density values 277 are in close agreement to other coronaviruses [26]. The bending stiffness of lipid bilayers ranges between $2786.16-18.5 \mathrm{kCal} \cdot \mathrm{mol}^{-1}\left(10-30 k_{B} T\right)$ 27 29 . Here, following previous works, we adopt an average value of $27912.3 \mathrm{kCal} \cdot \mathrm{mol}^{-1}\left(20 k_{B} T\right)[14,30$.

280 The density of ACE2 receptors on the cell membrane, at thermodynamic equilibrium, is $\xi_{R}$ from which 281 we compute the dimensionless ratio $\tilde{\xi}=\frac{\xi_{R}}{\xi_{S}}$. Given the limited data available on the density of receptors ${ }_{282}$ on human cells $\xi_{R}$ is difficult to estimate, in particular because receptor density varies across cell types and 283 depends on the specific receptor. Moreover, no specific data is available (to the author's knowledge) about 284 the density of ACE2 in epithelial cells in human lungs, the target of SARS-CoV-2.

285 Chen et al. 31 measured a density of $480-640 \mu \mathrm{m}^{-2}$ for receptor of various species, while Damioli et al. 28632 measured a density of $\sim 4.8 \mu \mathrm{m}^{-2}$ for VEGFR2 receptors. Based on these measurements, we estimated ${ }_{287} \tilde{\xi}$ to vary between $0.1-0.0001$ and adopt these values in our simulations. The receptor diffusivity was taken 288 as $D=0.01 \mu \mathrm{m}^{2} \cdot \mathrm{s}^{-1}$ an average value for most cells [14, 30, 32]. Medicine 382, 727-733 (2020). 
341 [26] Neuman, B. W. et al. A structural analysis of M protein in coronavirus assembly and morphology. Journal of $342 \quad$ Structural Biology 174, 11-22 (2011).

343 [27] Lipowsky, R. \& Sackmann, E. Structure and dynamics of membranes: I. from cells to vesicles/II. generic and $344 \quad$ specific interactions (Elsevier, 1995).

345 [28] Marsh, D. Intrinsic curvature in normal and inverted lipid structures and in membranes. Biophysical journal $346 \quad$ 70, 2248-2255 (1996).

347 [29] Helfrich, W. Elastic Properties of Lipid Bilayers: Theory and Possible Experiments. Zeitschrift für Natur$348 \quad$ forschung $C$ 28, 693-703 (1973).

349 [30] Tzlil, S., Deserno, M., Gelbart, W. M. \& Ben-Shaul, A. A statistical-thermodynamic model of viral budding. 350

Walls, A. C. et al. Structure, Function, and Antigenicity of the SARS-CoV-2 Spike Glycoprotein. Cell 181, 281-292.e6 (2020). by the viral spike protein. Viruses 4, 1011-1033 (2012). 581, 215-220 (2020).

] Wrapp, D. et al. Cryo-EM structure of the 2019-nCoV spike in the prefusion conformation. Science 367, 1260-1263 (2020)

7] Smith, M. \& Smith, J. C. Repurposing Therapeutics for COVID-19: Supercomputer-Based Docking to the SARS-CoV-2 Viral Spike Protein and Viral Spike Protein-Human ACE2 Interface. Preprint at 10.26434/chem(2020) Motif the Hallmark for Its Enhanced Infectivity? Insights from All-Atom Simulations. The Journal of Physical Chemistry Letters 11, 4785-4790 (2020).

9] He, J., Tao, H., Yan, Y., Huang, S.-Y. \& Xiao, Y. Molecular Mechanism of Evolution and Human Infection with SARS-CoV-2. Viruses 12, 428 (2020). acting with the ACE2 Human Receptor. Viruses 12, 497 (2020). SARS-CoV-2 and SARS-CoV. The Journal of Physical Chemistry Letters 11, 4897-4900 (2020). Advances 6 (2020).

13] Evans, E. A. \& Calderwood, D. A. Forces and Bond Dynamics in Cell Adhesion. Science 316, 1148-1153 (2007). Academy of Sciences 102, 9469-9474 (2005).

5] Bar-On, Y. M., Flamholz, A., Phillips, R. \& Milo, R. Science Forum: SARS-CoV-2 (COVID-19) by the numbers. eLife 9, e57309 (2020). dynamics implementation. Computer Physics Communications 91, 43-56 (1995). analysis. Molecular modeling annual 7, 306-317 (2001). laptops to supercomputers. SoftwareX 1-2, 19-25 (2015). 894-904.e9 (2020). parison to NMR data. Journal of computational chemistry 34, 2135-2145 (2013). with coupling to an external bath. The Journal of Chemical Physics 81, 3684-3690 (1984). (1995) and Computation 4, 116-122 (2008). Including Robust Error and Autocorrelation Estimates. Journal of Chemical Theory and Computation 6, 37133720 (2010).

Biophysical journal 86, 2037-2048 (2004). 
351 [31] Chen, Y. et al. Mapping receptor density on live cells by using fluorescence correlation spectroscopy. Chemistry $352 \quad-$ A European Journal 15, 5327-5336 (2009).

353 [32] Damioli, V., Salvadori, A., Beretta, G. P., Ravelli, C. \& Mitola, S. Multi-physics interactions drive VEGFR2 354 relocation on endothelial cells. Scientific Reports 7, 16700 (2017). 


\section{Figures}

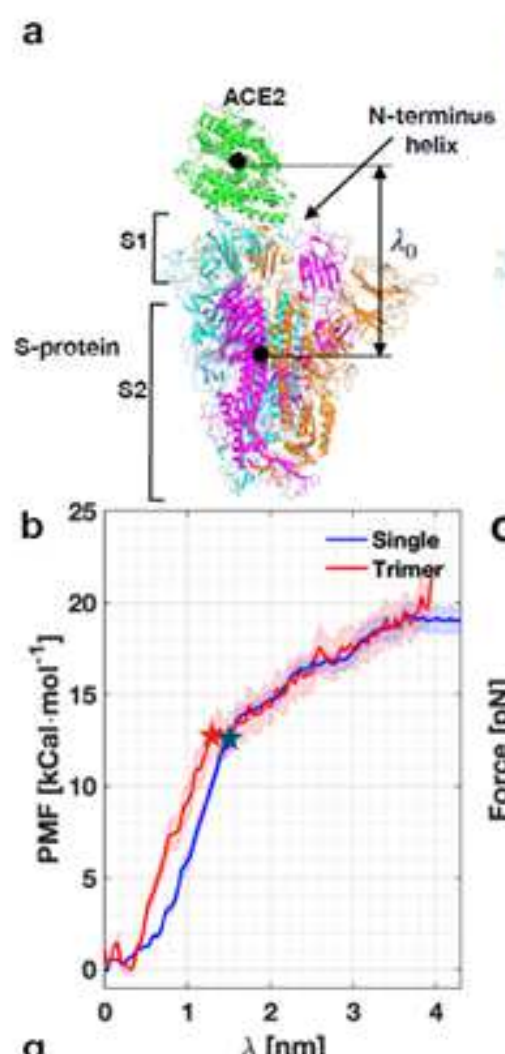

$$
9
$$
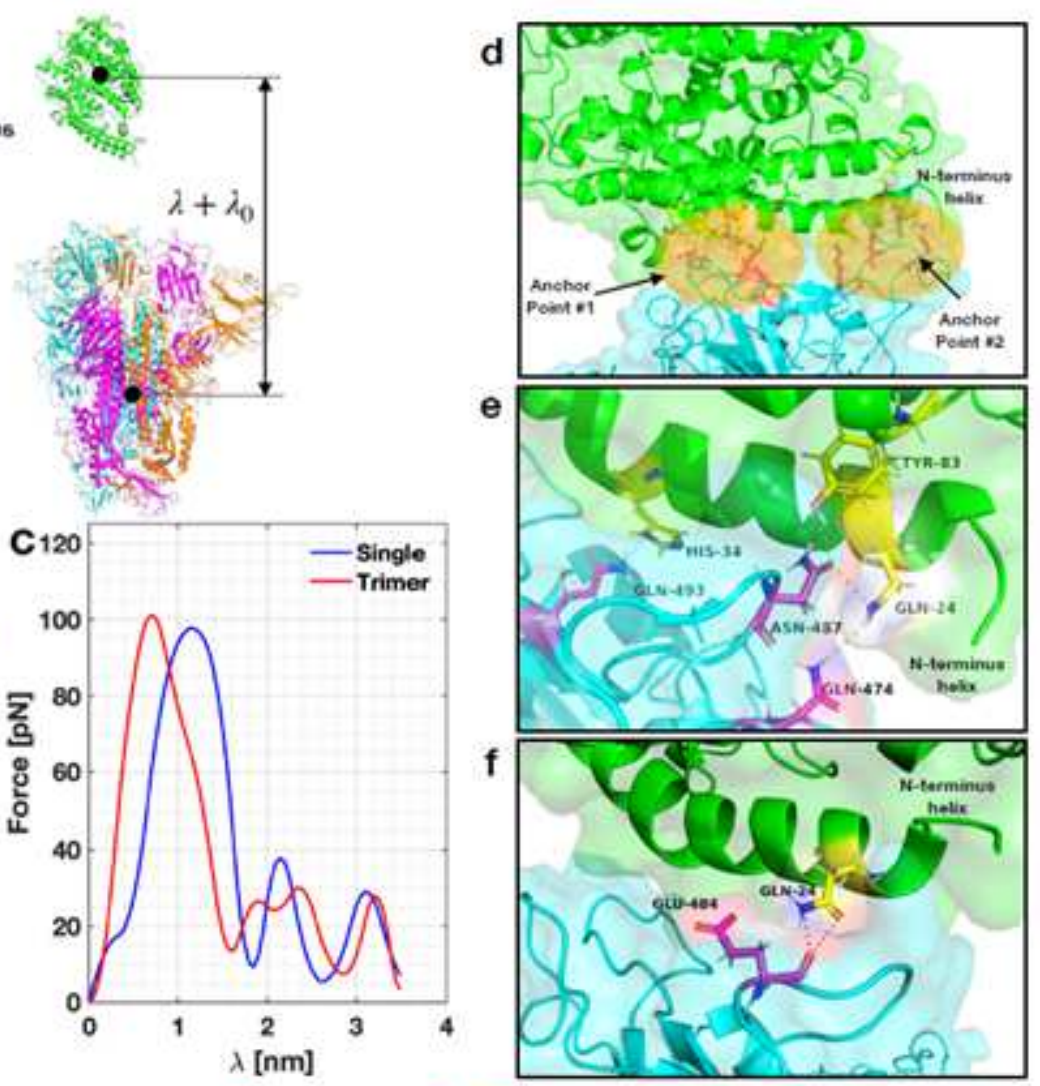

445 ... GGNYNYLYRLFRKSNLKPFERDISTEIYQAGSTPCNGVEGFNCYFPLOSYGFQPTNGVGY - 506

\section{Figure 1}

Evolution of the PMF, force, and residues with $\lambda$. (a) Schematics of the setup. (b) and (c) Evolution of the PMF and force as a function of $\lambda$ for a single S-protein/ACE2 receptor (blue) and for the trimeric protein (red). Shaded area in (b) represents the error band for both simulations. The stars indicate the moment when detachment has happened. (d) View of the two anchor points between S-protein/ACE2 receptor when $\lambda=0: 3 \mathrm{~nm}$. (e) Interacting residues for $\lambda=0: 8 \mathrm{~nm}$ and (f) $\lambda=1: 4 \mathrm{~nm}$. (g) List of residues that are active during the pulling simulation in the RBD. Letters in red, cyan background, bold and underlined correspond to residues in contact at $\lambda=\{0: 3 ; 0: 4 ; 0: 8 ; 1: 4\}$, respectively. Red dots indicate residues identified experimentally by Lan et al. [4]. Residue K-417 has been omitted in the sequence for shortness. 


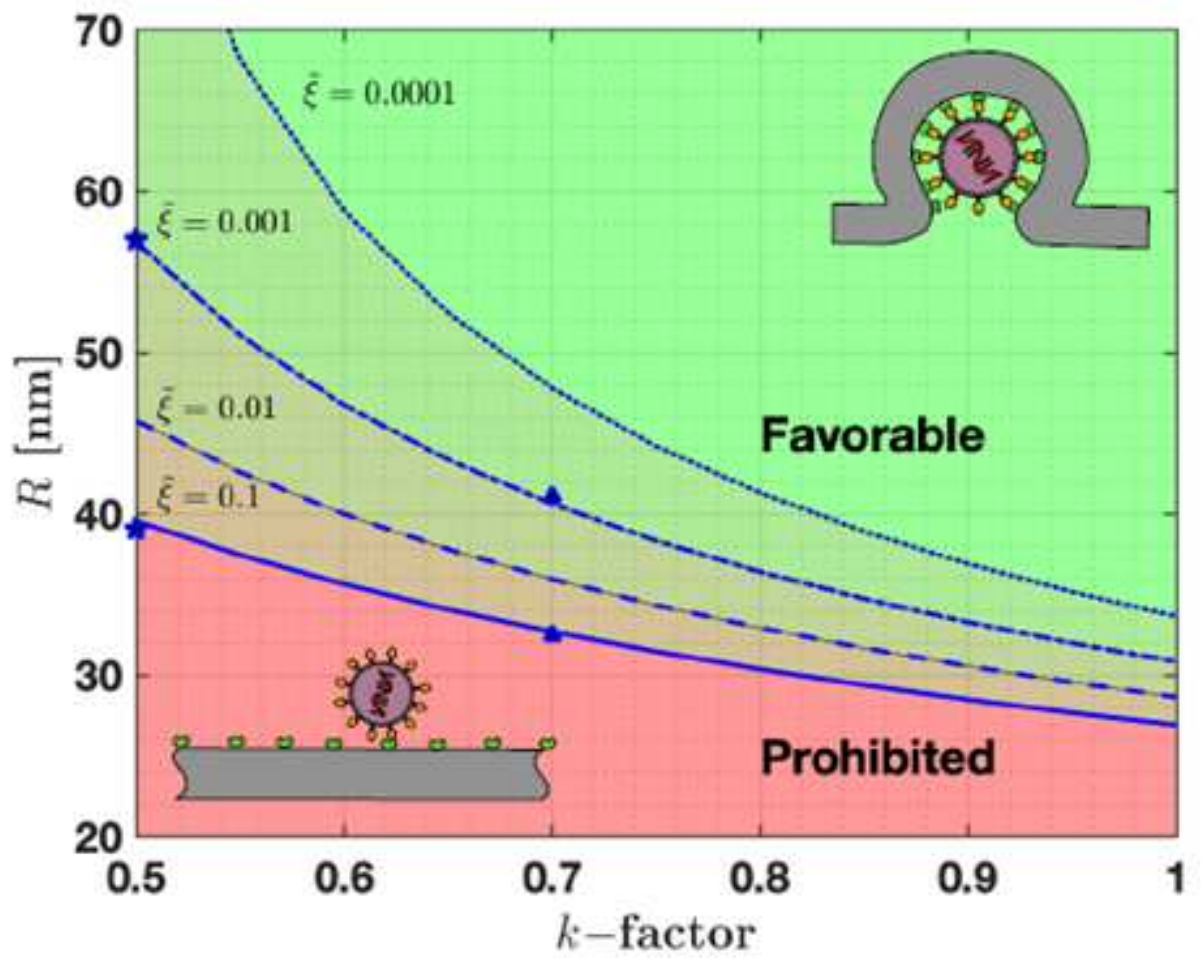

Figure 2

Effect of reduced binding affinity ( $k$-factor) in the size of particles that can be wrapped to the cell membrane. Four receptor densities were used, namely $\sim \xi=0: 1$ (solid line), $\sim \xi=0: 01$ (dashed line), $\sim \xi=$ 0:001 (dashed-dotted line), $\sim \xi=0: 0001$ (dotted line). Prohibited particles are in the red zone, while favorable particles are shown with in green. In between these zones, particle will be blocked depending on the relative receptor/S-proteins density $\sim \xi$.

\section{Supplementary Files}

This is a list of supplementary files associated with this preprint. Click to download.

- VideoSI1.mov

- VideoSI2.mov

- SI.pdf 